\title{
Las aves como naturaleza y la conservación de las aves como cultura ${ }^{1}$
}

\section{Joan David Tàbara}

Universitat Autònoma de Barcelona. Institut de Ciència i Tecnologia Ambiental (ICTA)

08193 Bellaterra (Barcelona). Spain

joandavid.tabara@uab.es

\section{Resumen}

El artículo argumenta que el proyecto internacional entorno al movimiento de conservación de las aves puede llegar a servir de gran ayuda, para enfrentarse con algunos de los más importantes límites culturales a la sostenibilidad, si bien puede hacerse a través de medios diferentes a los puramente científicos o racionales. Históricamente, el desarrollo del movimiento ambientalista estuvo, y aún está, muy vinculado a la utilización estratégica de las aves silvestres como palanca de sus reivindicaciones. Por un lado, los pájaros silvestres han ayudado a hacer explícito y visible el impacto de las poblaciones humanas sobre el medio ambiente y, por otro, han contribuido a concienciar a amplios sectores de la sociedad sobre la necesidad de adoptar compromisos con diversas políticas de carácter medioambiental. Las aves como naturaleza y la conservación de aves como cultura constituyen dos de las relaciones globales más exitosas de las culturas occidentales en la reunificación selectiva, reevaluación y comprensión de la naturaleza. Por una parte, la presencia de aves y de hábitats adecuados para ellas son utilizados como indicadores científicos de la calidad ambiental (naturaleza). Por otra, el nivel de protección de la avifauna en un país funciona como un punto de referencia del grado de «conciencia ambiental», de «responsabilidad moral» y de «civilización» (cultura). Las aves se han convertido en uno de los nuevos híbridos más sobresalientes entre «cultura y naturaleza» dentro de los discursos globales actuales sobre el medio ambiente, el cual está principalmente construido a través de los medios de comunicación de masas.

Palabras clave: conservación de los pájaros, límites culturales a la sostenibilidad, cambio ambiental, híbridos entre cultura y naturaleza, ornitología, ciencia popular.

\section{Abstract. Birds as nature, bird conservation as culture}

This paper argues that the project of the international bird conservation movement may help, by means other than purely scientific or rational, to deal decisively with some of the most important cultural limits to sustainability. Historically, the emergence and development of the environmental movement is very much linked to the strategic use of wild birds in

1. El presente artículo fue presentado originariamente en la conferencia del Grupo de Investigación 24 Sociedad y Medio Ambiente, de la Asociación Internacional de Sociología New Natures, New Cultures, New Technologies, celebrada en julio de 2001 en el Fitzwilliam College, Cambridge University, Reino Unido, bajo el título «Birds as nature, and bird conservation as culture». Agradezco al Dr. José Manuel Echavarren la presente traducción. 
revealing the impact of human populations upon the environment and in engaging large sectors of the population in the support of a variety of environmental policies. Birds as nature and bird conservation as culture constitute two of the most successful global social relationships of Western cultures in the selective reunification, re-valuation and understanding of nature. On the one hand, bird presence and adequate bird habitats are used as a scientific indicator of environmental quality (nature). On the other, the level of wild bird protection in a given country functions as a benchmark of the degree of environmental "conscience», "moral responsibility» and "civilisation» (culture). Birds have become some of the most prominent new "culture-nature» hybrids in contemporary mass mediated global discourses which construct our ideas about the environment.

Key words: bird conservation, cultural limits to sustainability, environmental change, hybrids culture-nature, ornithology, popular science.

\section{Sumario}

\section{Introducción 4. Las aves salvajes.}

2. Aves como naturaleza.

Un proyecto de la ciencia

3. La conservación de aves como cultura. Un proyecto de no expertos
¿Una nueva "cultura-naturaleza» global?

\section{Conclusión}

Bibliografía

He who shall hurt the little Wren,

Shall never be beloved by $\mathrm{Men}^{2}$.

(William Blake, Auguries of Innocence)

\section{Introducción}

En el universo social, existen tantas naturalezas como relaciones sociales con la naturaleza. Dicho de manera más específica, se pueden encontrar tantas concepciones y realidades acerca del mundo natural, como relaciones sociales imbricadas con un conjunto de objetos externos reificados y agrupados bajo la nomenclatura de naturaleza. Conforme van cambiando las relaciones sociales, el contenido asociado a los objetos naturales se va transformando, al igual que los significados culturales y los descubrimientos científicos que se logran extraer a partir de ellos. Naturaleza y cultura evolucionan de forma conjunta, o al menos los espacios de la naturaleza y de la cultura que conocemos. De esta forma, la "naturaleza» no existe hasta que se desarrolla una relación social que se construye a partir de una identificación, seguida por una extracción distintiva, de aquella realidad dada, interpretada como un elemento separado de la esfera humana, ajeno a ella. De modo similar a lo que sucede con respecto a otras

2. «Aquél quien al pájaro reyezuelo dañe, jamás querido por el hombre será». 
relaciones sociales, una vez que se ha llevado a cabo esa separación e identificación, la constitución original tanto del sujeto como del objeto que conforman la relación entre lo social y lo natural, queda abierta a procesos de interpretación desde otras perspectivas, así como a dinámicas de transformación. Entonces, el objeto llega a formar parte de la producción del sujeto y viceversa.

El presente texto, sin embargo, no intenta explorar todas las relaciones sociales con la naturaleza en todos sus objetos y formas. En lugar de explorar la "naturaleza» como una entidad total o en general, aquí se va a abordar esta problemática desde un ángulo metodológico diferente. Aquí nos vamos a concentrar únicamente en el estudio de un particular objeto de naturaleza aparentemente sencillo y no problematizado, los pájaros, y en dos de las relaciones exitosas con respecto a las aves en las sociedades occidentales contemporáneas: las aves como objeto de conocimiento científico y las aves como sujetos de conservación. Por una parte, las ciencias naturales, y dentro de ellas, la ornitología en particular, han sido decisivas en el proceso de definición, separación, y categorización de estas especies del reino animal, construyéndolas como un elemento distinto e independiente del mundo del ser humano. Por otro lado, el movimiento de conservación de las aves, que se desarrolló de forma conjunta pero también en oposición a muchos de los principios fundamentales esgrimidos por la comunidad científica, desarrolló un enfoque diferente, caracterizado por una reunificación moral y estética, que contrastaba con la mantenida por los ornitólogos profesionales. Como se verá a continuación, estas dos relaciones sociales se están fundiendo en las actuales sociedades contemporáneas, plenamente mediatizadas por los medios de comunicación de masas, en la producción de la nueva cultura-naturaleza híbrida universal de las aves.

Pero el centro de mayor interés aquí no es el análisis del papel que los pájaros han llevado a cabo en el descubrimiento de las realidades ecológicas científicas, sino más bien el estudio del tipo de actitudes, significados y funciones que se proyectan en esta clase de animales, tanto por la ciencia como por el público lego. Aunque voy a exponer brevemente los orígenes y el desarrollo de la interpretación particular y los usos de los pájaros por parte de la academia científica, el presente texto se centra mayormente en las nuevas relaciones sociales emergentes - percepciones por parte del público general, así como las interpretaciones y usos- con el mundo avícola «más allá» del discurso científico. A la hora de profundizar sobre las razones de la creciente relevancia cultural de la que disfrutan tales objetos naturales animados (con una dinámica propia e independiente de los humanos), se tiene la intención de proporcionar un marco teórico para una mejor comprensión del éxito de otras poderosas definiciones y afirmaciones contemporáneas al respecto de la naturaleza en las sociedades actuales, mayoritariamente urbanas. Este enfoque puede también ser relevante para comprender, en parte, las implicaciones de orden político, económico y medioambiental de la globalización de una forma cultural concreta de acercarse a la naturaleza, así como para entender la resistencia que ésta misma genera en algunas sociedades ajenas al ámbito anglosajón. El material 
empírico que se ofrece en el presente texto es fruto del análisis de contenido de fuentes publicadas principalmente en Estados Unidos, Gran Bretaña y España, así como de las experiencias personales de más de una década de observación participante en reservas naturales para aves y en grupos conservacionistas en los dos países europeos anteriormente citados.

\section{Aves como naturaleza. Un proyecto de la ciencia}

De acuerdo con la interpretación clásica de Edwin Stresemann, probablemente fue Aristóteles (384-322 aC), en su Historia de los animales, el primer pensador en elevar el "conocimiento de los pájaros a la categoría de ciencia». Para Stresemann, esta interpretación del conocimiento sobre avifauna como una rama del conocimiento científico, se pudo desarrollar como tal gracias a la capacidad de describir y separar los diferentes grupos de aves, sus hábitos, necesidades, anatomía, así como de indicar algunos de los principios básicos de organización de las distintas categorías de pájaros. De mayor relevancia, sin embargo, era la manera de acercarse al estudio de las aves, lo que revelaba la perspectiva científica de Aristóteles al respecto. Para este autor, el investigador del mundo animal debía tratar de establecer la relación del fenómeno en cuestión con el propósito inherente (o razón de ser) para el cual existía cada criatura viva, esto es, independientemente del significado que los humanos le atribuían $^{3}$.

Es precisamente en esta separación entre el observador y el observado, en nuestro caso entre los seres humanos y las aves, donde subyace el origen de la ornitología como ciencia ${ }^{4}$. Esta posición, sin embargo, se dejó de lado durante un largo periodo de tiempo, y con contadas excepciones, hasta el Renacimiento. Durante la edad media, las descripciones de pájaros estaban tamizadas por la religión y por las aportaciones de la tradición popular, de modo que los pocos avances en el conocimiento de los pájaros se dieron tan sólo en campos como la cetrería y el manejo de la caza con aves. No fue hasta el siglo XVI cuando se dio un nuevo empuje científico a la disciplina, con trabajos como la Historia de la naturaleza de las aves, del francés Pierre Belon (1517-1564), y el Tratado de Zoología, del alemán Honrad Gessner (15161565). En el siglo XVIII, la confluencia de varios desarrollos científicos, principalmente la sistemática, la penetración de nuevas teorías y de conceptos, el incremento en el número de observadores que se dedicaban a reunir muestras y materiales de todo tipo, así como la importancia creciente de los exploradores, proporcionó a la ornitología la oportunidad de irse diferenciando, como rama distinta, de la zoología. Fue aquélla una época de importantes expediciones de carácter científico, de la publicación de descripciones detalladas de avifauna regional y de descubrimientos de gran relevancia en materia de anato-

3. E. Streseman (1975: 3-7).

4. Consultar también, a este respecto, el enfoque más generalista de N. Evernden (1992). 
mía y el comportamiento lo que terminó por conducir, en el siglo XX, a un nuevo interés en la ecología, la etología y la adaptación funcional de las aves ${ }^{5}$.

Los avances científicos en el estudio de las aves se vieron favorecidos por el desarrollo de nuevas técnicas y herramientas para capturar, preservar y examinar pájaros. Sin embargo, la mayoría de los métodos "científicos» de la disciplina a fines del siglo XIX, tales como la caza o la captura de huevos, y en particular de aves exóticas, difícilmente podían ayudar a establecer lazos emocionales con el mundo de las aves. Como quedaba reflejado en un popular manual estadounidense para estudiosos de las aves, escrito por John Burroughs:

En primer lugar, debe localizar al ave en cuestión; observar sus conductas, sus cantos, sus reclamos, sus vuelos, sus guaridas; después, dispárele (y deje de echarle miradas amorosas a través de los prismáticos), y compárese con Audubon. De esta forma, el reino alado pronto será conquistado. (J. Burroughs, 1877, citado en M. V. Barrow Jr. 1998: 113) ${ }^{6}$

A fines del siglo XIX, el debate suscitado para detener el uso de estos métodos científicos, que estaban innegablemente afectando al destino de algunas especies en peligro de extinción, originó considerables conflictos dentro de la comunidad ornitológica. El eje del debate no trataba meramente acerca de detener la pretensión de los científicos de comprender el mundo natural. Más bien, las discusiones, de carácter más profundo, eran resultado de confrontaciones culturales y, de forma más amplia, metafísicas, acerca de si era necesario permanecer separado de la naturaleza, pretender dominarla o, incluso, si tenía algún sentido el plantearse todo esto siquiera, a fin de comprenderla.

Muchas de aquellas actitudes, que entendían a las aves como objetos naturales separados del reino de lo humano, continuaron y se extendieron dentro de la comunidad ornitológica incluso hasta tiempos presentes. Actualmente, se tiende a evitar el uso de la mayoría de los métodos "científicos» con efectos irreversibles sobre especies concretas, a la vez que entran en liza otras técnicas más sofisticadas. El rastreo por radio, el anillado y etiquetado de aves, llega en algunos casos en Europa hasta alcanzar los varios centenares de miles de pájaros, los cuales se emplean normalmente para estudiar los movimientos de aves y muchas otras facetas de la ecología de estos tipos de animales y sus conductas. Como señaló un ornitólogo y anillador de aves español, en una conferencia ante el Institut Català d'Ornitologia de Barcelona:

El anillado de aves se justifica éticamente porque aumenta el conocimiento. (J. M. C., Barcelona, 1997)

5. Ver Farber, P. L. (1997) y J. Maluquer i Sostres (1986-1993).

6. Tomado del libro de J. Burroughs, Wake-Robin (Houghton, Mifflin, 1877: 231), citado en M. V. Barrow Jr. (1998: 113). Sin embargo, más adelante, Burroughs tomó posturas muy críticas y fue uno de los defensores más activos de cambios drásticos en los métodos tradicionales utilizados por los ornitólogos. 
De este modo, la ciencia y la ornitología en particular han desarrollado una relación social con este objeto de la naturaleza en una forma donde el observador permanece a distancia de lo observado y donde las aves se interpretan meramente como una fuente de conocimiento. Recordando las palabras del antiguo director del Museo de Zoología de Barcelona, y también prominente ornitólogo profesional, al hilo de la reflexión sobre un estudio de aves:

Lo que es importante es el concepto, no las especies. (F. O., Barcelona, 1996)

Sin embargo, y en franco contraste con otras disciplinas de las ciencias naturales, el proyecto de la ornitología de obtener un creciente conocimiento "objetivo» no podría sostenerse sin la ayuda de un cuantioso número de personas conocedoras pero no expertas, principalmente, entusiastas de las aves sin pretensiones de hacer una profesión de su pasatiempo. En muchos aspectos, la ornitología ha sido, y no puede ser de otro modo, siempre una «ciencia popular», a pesar de sus muchos y repetidos intentos de convertirse en una ciencia de laboratorio como la química o la física. A pesar de ello, y especialmente después de que esta disciplina comenzara a ganar reconocimiento y a alcanzar el estatus profesional durante el siglo XIX, las relaciones entre los profesionales y los amateurs se fueron haciendo más problemáticas. Mientras los primeros a menudo veían amenazada su autoridad científica por la presencia numerosa de éstos últimos (por ejemplo, en las publicaciones especializadas), los científicos y los profesionales de la avifauna no podían, sin embargo, desarrollar su trabajo sin el apoyo de los amateurs. A pesar de los avances y de la sofisticación de los métodos de la ciencia de las aves, la colaboración con la nueva multitud de observadores de pájaros, conservacionistas y «entusiastas de las aves», tenía que ser continuamente aceptada. El interés de la población no experta contribuyó decisivamente a la producción de datos y a reunir los fondos necesarios para emprender las actividades de investigación más reservadas y más «elitistas». La interdependencia que existía entre la ornitología y los entusiastas convirtió a esta disciplina en algo mucho más abierto al lenguaje popular y a la participación pública. Con ello, se emprendieron nuevas vías para establecer nuevas prioridades de investigación y nuevos programas, más cercanos a las demandas del público general, y no solamente enfocados a la obtención de "conocimiento». Esta interpenetración no ha sucedido en otras ciencias naturales disciplinares, mucho más afianzadas en sus complejas metodologías especializadas y menos dispuestas a permitir interferencias por parte de no expertos, aunque es paradigmática de las ciencias ambientales aplicadas en general 7 .

7. Consultar M. V., Jr. Barrow (1998), y J.M. Drouin y B. Bensaude-Vinvent (1996). 


\section{La conservación de aves como cultura. Un proyecto de no expertos ${ }^{8}$}

La colaboración de los ornitólogos profesionales con el incipiente movimiento conservacionista no solamente tuvo como consecuencia frecuentes tensiones internas dentro de la comunidad científica que estudiaba las aves, sino que también influyó en su dirección, métodos y prioridades. Los ornitólogos lucharon durante todo un siglo para que se reconociera su disciplina, aunque ese reconocimiento tan sólo se alcanzara gracias al apoyo y a las alianzas establecidas con los amateurs y los entusiastas de las aves. Mientras los ornitólogos se esforzaban por desarrollar una disciplina equipada con métodos «objetivos» para estudiar las realidades «objetivas» de un aspecto particular del mundo natural, al mismo tiempo, una comunidad mucho más numerosa de población no experta estaba interesada en desarrollar una relación social ciertamente diferente con el mismo objeto de estudio. Lo que los observadores de pájaros y los entusiastas de las aves estaban buscando en el campo, no eran conceptos ni leyes, sino otro tipo de experiencias y significados. Más bien, la recreación de placeres estéticos e ideales sociales acerca de la naturaleza y la búsqueda de armonías entre los mundos natural y humano se convertirían en uno de los temas más prominentes en los discursos y en las prácticas de conservación de la avifauna.

Un caso paradigmático de cómo llegaron a ser esos conflictos y la tensa colaboración entre ambos colectivos se puede encontrar en el desarrollo del movimiento conservacionista en Estados Unidos. En dicho país, el movimiento de conservación de aves comenzó en la década de 1880, a partir de una amplia amalgama de intereses de grupos aparentemente diversos unidos por su oposición a la caza comercial a gran escala, tanto para la obtención de carne como de plumas. Entre todas aquellas personas, se podían encontrar amantes de la naturaleza, organizaciones recién fundadas de prevención de crueldad contra los animales, así como cazadores y algunos científicos ${ }^{9}$. La creciente penetración de conceptos científicos en el movimiento de conservación de las aves proporcionó a sus argumentos un peso específico adicional. Nociones como la integridad del ecosistema y los efectos ecológicos de la extinción de ciertos predadores estaban ya bastante extendidas al comienzo del siglo XX. Las leyes de protección de aves, que originalmente comprendían tan sólo a las especies que se creían beneficiosas o que se caracterizaban por un notorio valor económico como piezas de caza o para la agricultura, se iban extendiendo. Las nuevas leyes llegarían a abarcar a aquellas aves amenazadas bajo la sombra de la extinción, así como a aquéllas que se intuía que podían llevar a cabo algún tipo de función reguladora en la conservación integral de los sistemas ecológicos.

8. En el presente texto, cuando se habla de aves y de conservación de aves, nos referimos a aves silvestres.

9. La sociedad Audubon, por ejemplo, se creó en 1886 con el objetivo de «proteger a las aves silvestres y sus huevos». 
Estos nuevos conceptos también se aprovecharon del nuevo clima cultural, el cual sostenía interpretaciones más amplias sobre los derechos de la naturaleza. La extensión de atribuciones morales hacia animales no humanos acabaría desembocando en ideas como que toda la vida salvaje debería poder disfrutar de un espacio propio donde "pudieran perseguir libremente sus propias formas de felicidad " ${ }^{10}$. Estas perspectivas sirvieron de inspiración a nuevas políticas de conservación que iban más allá de los meros intereses de recreo humanos, como fue el caso de la primera unidad de Refugio Nacional del Sistema de Vida Salvaje en los Estados Unidos, en la isla Pelican, en Florida, y que Theodore Roosevelt declaró, en 1903, como un espacio para "la preservación y el apareamiento de aves autóctonas» ${ }^{11}$. De hecho, Roosevelt, el primer cargo presidencial que tomó después de serlo de los Estados Unidos, fue el de presidente del club de ornitología de Long Island ${ }^{12}$.

Como Robin W. Doughty había señalado acertadamente, algunos de aquellos primeros intentos no científicos de promover el interés del público general en la lucha por la conservación de las aves, tenían como objetivo el cambiar las percepciones sobre los gustos. Por ejemplo, de lo que se trataba era intentar hacer pasar moda el hábito de llevar plumas en los sombreros, y representarlo como un acto depravado y cruel $^{13}$. En esta empresa, el activismo feminista jugó un papel muy importante. Las mujeres proporcionaron toda una nueva suerte de argumentos para la conservación de las aves que resultaban difíciles de ser enunciadas bajo los valores masculinos dominantes de la ornitología y de las sociedades de cazadores de la época. Para las mujeres como la bostoniana Harriet Hemenway, que, de acuerdo con Joseph Kastner, debería ser considerada por los observadores de aves como su santa matrona ${ }^{14}$, matar pájaros silvestres no sólo debería considerarse ilegal sino también éticamente inmoral. Las aves silvestres deberían ser consideradas como un objeto de respeto merecedor de protección simplemente porque se trataba de unas criaturas hermosas y adorables, porque sus canciones tenían la facultad de alegrar a las personas, y no tan sólo porque su extinción sería una pérdida irreversible para el progreso de la ciencia o los ecosistemas.

De esta forma, se puede distinguir, en las sociedades occidentales, el desarrollo de dos tipos ideales de relaciones culturales con el objeto natural de las aves. Por un lado, una ciencia de la ornitología dominada (y dominante) originalmente por el género masculino, afincada principalmente en los museos, en las publicaciones científicas y en los debates académicos. Y, por otro lado, otra comunidad más abierta, más amplia, dinámica y plural, donde la participa-

10. R. F. Nash (1989: 170).

11. Ibídem, 171.

12. J. Hill (1999).

13. R. W. Doughty (1975).

14. J. Kastner (1994: 104). La primera presidencia de la Royal Society for the Protection of Birds británica fue ocupada por una mujer, y durante 65 años, hasta 1954, la condesa de Portland, Winifred (Hill, 1999). 


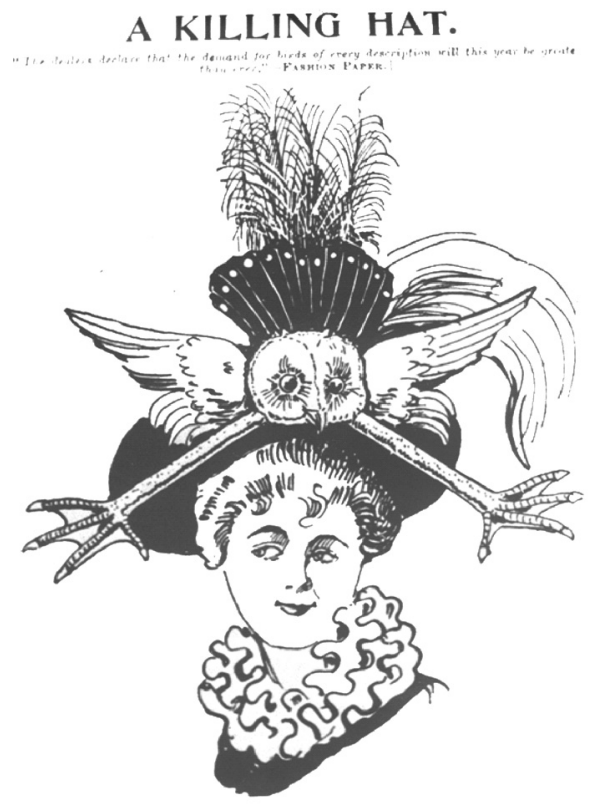

Figura 1. Caricatura de 1910 de la Westminster Gazette, y reimpresa por la Royal Society for the Protection of Birds británica para denunciar el mal gusto y la moda de llevar plumas de pájaros en el sombrero. Mientras que en un lugar se denunciaba que la demanda de plumas, según los comerciantes, sería aquel año mayor que nunca, la caricatura se acompañaba con el siguiente texto: «He encontrado un regalo para mi feria: un par de patas de cigüeña — piénsalo! Si ciertamente se ven absurdas, es culpa del pájaro, por no hacer crecer más patas que vayan bien para el sombrero». Fuente: Doughty (1975: 82).

ción de las mujeres probó ser decisiva, y que tenía más interés en promover un tipo de asociación social diferente con las aves. Principalmente, la nueva perspectiva iba a promover los nuevos modos del conservacionismo, basados en la «reunificación» entre el hombre y la naturaleza (ya seleccionada) e incluso el redescubrimiento de una «hermandad animal» ${ }^{15}$. Compromisos normativos de una particular concepción de prácticas profesionales y de autoimáge-

15. Como señala Lawrence Buell (1995), «una de las aportaciones dramáticas en el pensamiento postromántico al respecto de la naturaleza ha sido el declive y el resurgimiento de la "hermandad" entre lo no-humano y lo humano. Su raíz metafísica cobró fuerza en la última mitad del siglo XIX; el modernismo anunció su muerte; el ecologismo moderno lo ha recuperado de nuevo. No obstante, el "avifaunismo" no puede entenderse como una simple expresión del antropomorfismo, dado que gran parte de la experiencia cognitiva y emocional que deriva de la observación y de la conservación de aves en libertad reside precisamente en el mantenimiento de los pájaros en libertad como "fuera" de la esfera de lo humano» (véase R. W. Mitchell y otros, 1997). 
nes rígidas, impulsaron a la mayoría de los ornitólogos a desechar los argumentos acerca de las cuestiones conservacionistas que no estuvieran directamente relacionadas con razones de utilidad científica o puro avance del conocimiento. Con todo, la comunidad de conservacionistas era libre de expresar su preocupación acerca de la matanza de aves y la destrucción de sus hábitats, sobre la base de la belleza, del gusto y de otros factores emocionales. La academia científica no podía expresar abiertamente su "amor por la naturaleza», y los amantes de los pájaros no podían, a su vez, justificar sus sentimientos únicamente a partir de razones puramente utilitarias o científicas. Para el amplio público entusiasta no experto, las aves silvestres y los hábitats naturales donde habitaban debían ser salvados, pero no principalmente porque contribuían al equilibrio de los sistemas ecológicos o porque pudieran perderse datos biológicos o fuentes de conocimiento. Simplemente, las aves debían ser protegidas porque resultaban hermosas de contemplar y de escuchar, merecían ser consideradas como sujetos morales, y quizás pudieran comunicar al público verdades acerca del mundo natural y del medio ambiente que otros objetos naturales pudieran no ser capaces de comunicar. Las limitaciones y la mutua interdependencia de las dos comunidades para sostener sus aspiraciones y las actividades de ambos hicieron que su colaboración, relativamente distante, pero al mismo tiempo cercana, pudiera ser la base del movimiento conservacionista de aves que existe en la actualidad y de la consolidación de la ornitología como ciencia.. Conforme las relaciones se intensificaron y el número de intereses se expandió en ambas comunidades, los científicos tomaron un creciente protagonismo en la defensa de las demandas civiles, y los conservacionistas fueron financiando con mayor interés las actividades de investigación de la academia científica. A pesar de todo, en la actualidad, todavía persisten las tensiones entre los dos proyectos.

\section{Las aves salvajes, ¿una nueva «cultura-naturaleza» global?}

El origen de muchos de los grupos medioambientales occidentales contemporáneos puede hallarse en el movimiento de protección de las aves. Como se ha señalado anteriormente, las primeras organizaciones conservacionistas anglosajonas se crearon a finales del siglo XIX como reacción a la caza de aves a gran escala, particularmente en los Estados Unidos. El mismo David Thoreau, uno de los grandes inspiradores del movimiento ambientalista americano, fue uno de los primeros autores en destacar la necesidad de fusión entre el conocimiento científico y la experiencia emocional, en la que la observación de pájaros tendría un lugar muy destacado ${ }^{16}$.

En el siglo XX, el renacimiento de una segunda ola de ambientalismo también está muy ligado a la «evidencia» de la destrucción medioambiental revelada por los pájaros y denunciada en trabajos como el de la Primavera silen- 
ciosa, de Rachel Carson ${ }^{17}$. El famoso libro de Carson relataba historias de aves envenenadas, como las del petirrojo, el pájaro trepador blanco, el peregrino o el águila calva, que sirvieron para dar la señal de alarma sobre los masivos efectos negativos sobre el medio ambiente que tenía el uso de pesticidas y otros «elixires» mortales como el DDT ${ }^{18}$. Ahora que el siglo XX ha quedado atrás, las aves han regresado de nuevo, desvelando nuevas realidades y significados medioambientales e, inevitablemente, abriéndose camino en los corazones de los seres humanos de formas diferentes y por razones diversas.

Hoy en día, pocos de entre nosotros pondríamos en cuestión que las aves son naturaleza, de hecho, una parte particularmente bella de la naturaleza. Esta apreciación es casi universal en las sociedades occidentales contemporáneas, y la utilización de aves, tanto en los medios de comunicación de masas como en numerosas estrategias educacionales para representar ejemplos vívidos de naturaleza salvaje, ha llegado a alcanzar proporciones globales. Las actividades de ocio relacionadas con los pájaros también han estado en creciente boga, en especial a partir de la segunda mitad del siglo XX y estimulado con las nuevas posibilidades de movilidad ${ }^{19}$. Por ejemplo, la Sociedad Audubon señalaba que, tan sólo en Canadá, Estados Unidos y México, la observación de aves genera veinticinco mil millones de dólares al año, mientras que industrias rivales como la química o la del acero, atraviesan una época de recesión ${ }^{20}$. Al otro lado del océano Atlántico, la Real Sociedad Británica para la Protección de Aves (RSPB) británica, engloba ya más de un millón de socios y cita que, al menos, se generan once millones de libras al año de beneficios en las economías locales como resultado de las visitas a las reservas de aves ${ }^{21}$. La observación de pájaros se está convirtiendo en una industria por derecho propio - educacional, turística, base para la planificación territorial—, que, a su vez, está contribuyendo a que numerosos sectores de la población occidental tengan una conciencia medioambiental más elevada. Este fenómeno suele comenzar, en primer lugar, cuando la población concentra su atención en algunas de las especies emblemáticas para, después, ir introduciendo otras muchas ideas de corte ecológico en la interpretación de sus propias vidas. Es por ello que podemos afirmar que el descubrimiento de un objeto clave de la naturaleza —en este caso, los pájaros- abre nuevas oportunidades para la redefinición y autocomprensión del sujeto - la sociedad.

De este modo, la conservación de aves también actúa como una poderosa justificación simbólica para otras muchas políticas medioambientales de

17. Aquí, el título, Silent Spring (Primavera silenciosa), hace referencia a un escenario primaveral sin el trinar de los pájaros. La imagen metafórica causó un gran impacto en el público norteamericano.

18. R. Carson (1962, passim). Como señala Shackleton (1962) en la introducción del libro de Carson, eran los «montones de cadáveres de pájaros los que señalaban la verdad» (p. XVI).

19. S. Moss (2004).

20. The Gallon Environment Letter (2001).

21. RSPB (2000 y s.). 
amplia repercusión, las cuales no habrían podido ser justificadas únicamente a partir de discursos puramente «racionales» o «científicos». Las asociaciones de conservación de aves trabajan, en la actualidad, activamente junto con una gran variedad de organizaciones de carácter privado y público, en campos tan diversos como la agricultura, la energía y el sector financiero. Las acciones orientadas hacia la protección de aves pueden ayudar a reconducir con éxito el crecimiento económico e incluso reducir ciertas prácticas de carácter no sostenible, tanto en los sectores de la producción como del consumo y, a su tiempo, justificar o comenzar procesos de innovación medioambiental. El sector agropecuario, por ejemplo, como el del arroz en el delta del río Ebro en Cataluña, están de hecho produciendo cosechas «respetuosas con las aves», como resultado de la colaboración entre asociaciones conservacionistas, científicos y grupos privados.

Además, el tema de la protección de las aves se utiliza, políticamente, como parte de un nuevo tipo de internacionalismo ecológico que tiene como objetivo modificar aquellas culturas con percepciones, valores y relaciones diferentes con este particular objeto de la naturaleza. En Europa meridional, por ejemplo, quinientos millones de pájaros son cazados en trampas ilegalmente cada año. En muchos de estos lugares, los pájaros silvestres se consideran como un manjar exquisito (un verderón macho puede alcanzar las cincuenta libras británicas en una taberna de Malta). Esta situación ha hecho que algunas organizaciones conservacionistas defendieran la no entrada de países como Malta o Chipre en la Unión Europea hasta que las leyes que limitan estas prácticas tradicionales de caza de aves se hubieran endurecido suficientemente. Sin embargo, la resistencia a alterar actitudes y prácticas culturales como éstas es elevada y revela el profundo contraste entre el sur y el norte europeos y los países anglosajones, en la forma como tratan a las aves en particular y a los animales en general. A pesar del avance hecho en varias directivas europeas y del número creciente de otras políticas de la Unión Europea en este campo ${ }^{22}$, la Unión Europea todavía no ha sido capaz de reforzar de manera suficientemente efectiva las leyes de protección de aves dentro de sus estados miembros, y menos aún más allá de sus fronteras. Ésta es la razón por la cual muchos conservacionistas del norte de Europa y entusiastas de las aves entienden que en la Unión Europea podría ocurrir un retroceso en este campo y señalan la necesidad de poner en marcha acciones de tipo inmediato. En palabras de una observadora de aves británica de la reserva de Cley, en Norfolk, Gran Bretaña:

No entiendo por qué esa gente de Bruselas no hace más por los pájaros. Seguramente, si la gente pudiera disfrutar más de las aves, sería mucho más feliz. (A. X, Norfolk, 2000)

22. Tales como la Directiva de Aves aprobada en 1979, la Directiva de Hábitats de 1992 y las futuras 2.400 áreas protegidas de la red Natura 2000. 
De acuerdo con muchos ambientalistas, las leyes no son suficientes para detener la masacre, ya que «únicamente la educación puede servir de algo» $(\text { ("cultura») })^{23}$.

Por tanto, se puede señalar, con razón, que la globalización creciente de esta forma de interpretar y de actuar con respecto a las aves silvestres, es, en su gran parte, el resultado de algunas percepciones socialmente exitosas sobre la naturaleza, entorno a la belleza, el gusto, la felicidad y los ideales relacionados con éstas ${ }^{24}$. Durante el siglo XVII, por ejemplo, «una colección de pájaros de vivos colores llegó a constituir el rasgo característico de todo jardín aristocrático» ${ }^{25}$. Los colonos europeos, por su lado, llevaron todo tipo de animales y plantas consigo a las nuevas tierras, prestando especial interés a las aves, no tan sólo como medio de controlar plagas, sino también como forma de recrear el hogar de su tierra natal ${ }^{26}$. Como señala el gran comunicador de la cadena de televisión británica BBC, David Attenbourgh, en uno de sus famosos trabajos y de impacto mundial sobre las aves, los colonos de Australia y Nueva Zelanda fundaron «sociedades de aclimatación» que incluían secciones dedicadas a «mejorar» la avifauna local. Las aves eran liberadas en Australia, de acuerdo con los deseos de estas sociedades, con el objetivo de que «se establezcan si es posible aquí y proporcionen a estas colinas y bosques un tanto silenciosos, la música y la armonía de la vida de la campiña inglesa» ${ }^{27}$.

Sin embargo, y a pesar de todo ello, cabe recordar que los pájaros no son solamente naturaleza. Hasta cierto punto, y a pesar de alguna resistencia cultural, las aves se están convirtiendo en los perfiles de reforma medioambiental en lo que Bruno Latour denomina cuasi objetos y cuasi sujetos. Los pájaros están «incluidos» en los discursos al respecto del medio ambiente, no tan sólo como objetos pasivos de la agencia humana, sino también como "agentes» activos naturales. Sus vidas y sus hábitos instintivos tienen la indiscutible capacidad de «informar» e incluso de «influir» — tanto científicamente como moralmente- en la voluntad humana. Los ejemplos son abundantes. En Gran

23. S. Papps (2001: 27). Hasta cierto punto, la situación actual en el sur europeo es comparable con la que tenía lugar en los Estados Unidos durante las últimas décadas del siglo XIX, cuando se cazaban varios cientos de millones de aves al año, fenómeno que desembocó en la extinción de algunas especies muy comunes, como la paloma pasajera.

24. Sin embargo, aunque quizás haya sido éste el caso desde épocas primitivas, la «globalización» y el uso de pájaros para cambiar las percepciones de las personas sobre el mundo puede haber comenzado en una fecha tan lejana como 1493, cuando Cristóbal Colón hizo su entrada ceremonial en Barcelona rodeado de loros de múltiples colores, a fin de impresionar al público y de proporcionar una prueba tangible del éxito de su empresa. A partir de ese momento, muchas otras aves provenientes de América se fueron introduciendo en Europa durante el siglo XVI (Stresseman, 1975: 24). Para consultar dos revisiones de la interpretación de la naturaleza en la literatura anglosajona, y en particular en Estados Unidos, ver L. Buell (1995) y L. Marx (1964).

25. K. Thomas (1983: 277).

26. Lo que, en algunos casos, tuvo devastadoras consecuencias en las poblaciones de aves indígenas. Ver A. Crosby (1986: 292-3).

27. Citado en Attenborough (1998: 300). 
Bretaña, la nidada de un halcón peregrino en una chimenea industrial desencadenó las demandas del público general para el cese de la actividad industrial dañina con el medio; en Barcelona, los planes de ampliar el aeropuerto cerca de una marisma rica en avifauna, se limitó en parte, ya que fue objeto de la oposición frontal por parte de los ecologistas; y en Estados Unidos, se han establecido fuertes argumentos a favor de la implementación de políticas para combatir el cambio climático basadas en el previsible impacto sobre las poblaciones nacionales de aves, con el fin de ganar más apoyo por parte del público. Asimismo, los pájaros son utilizados por los ecologistas y los amantes de la naturaleza como un instrumento para recordar concepciones alternativas del tiempo y del espacio, diferentes de aquéllas que enmarcan las decisiones de la sociedad monetaria industrial, basadas en la preeminencia del valor tiempo, por encima del valor espacio y/o naturaleza. Por una parte, la prueba de la existencia de un "tiempo natural» puede encontrarse en el calendario interno de las vidas de los pájaros con fases claramente definidas como la migración o la reproducción, mientras que, por otra parte, los bioregionalistas argumentan que los hábitats y la presencia de las aves es una de las maneras de reconocer una «región natural»" ${ }^{28}$. De esta forma, y como señala Latour, en la sociedad postmoderna actual, la separación de naturaleza y sociedad se hace cada vez más difícil de sostener, no tan sólo en el propio lenguaje hablado, en el ámbito intelectual y en el discurso, sino también en la vida cotidiana, debido a la penetración de «híbridos» que no son ni naturaleza ni cultura, sino las dos a la vez. Dice Latour sobre estos híbridos:

La bomba de aire de Boyle, los microbios de Pasteur, las poleas de Arquímedes, son los objetos de los que hablo. Estos nuevos no-humanos poseen propiedades milagrosas que son una y a la vez tanto sociales y asociales, productores de naturalezas y constructores de sujetos. (Latour, 1991: 112)

Las aves han sido reintroducidas, imperceptiblemente, no tan sólo en sus hábitats naturales, sino también como compañeros bióticos animados de nuestra cultura y en las decisiones de la gestión del paisaje urbano de nuestras ciudades. En una forma de proceso múltiple de postmodernización ecológica, las áreas de protección de aves y las políticas de conservación se están convirtiendo cada vez más en elementos centrales en los planes medioambientales de carácter local, las agendas 21 , así como en las políticas de calidad de aguas. Dado que un prerrequisito para el éxito de estas estrategias es un amplio proceso de participación pública, la práctica, un tanto informal aunque significativa, de ayudar a proteger o controlar la avifauna silvestre, hace a esta forma de contribución algo ciertamente atractivo y reconfortante. En gran medida,

28. En este trabajo no voy a profundizar en si estas posiciones son ontológicamente ciertas o no. Para esta empresa, sería necesaria una investigación mucho más extensa. Para un ejemplo original de la utilización de aves en discursos bioregionalistas, ver L. Charles y otros (1995). 
se puede decir que la conservación de aves se está convirtiendo en uno de los elementos más importantes dentro de la dimensión medioambiental del proceso de estructuración dual ${ }^{29}$ de las sociedades urbanas occidentales. Por un lado, la apreciación de las aves y la comprensión de sus funciones ecológicas han permitido la extensión actual de la percepción y sensibilidad humanas del entorno natural de una forma en la que algunas actividades humanas con efectos medioambientales irreversibles vayan a ir siendo eliminadas. En las áreas naturales remanentes ricas en avifauna, se han ido proyectando nuevos valores, de modo que las decisiones políticas y las actividades económicas ambientalmente dañinas se han ido evitando. Por otra parte, y a menudo al mismo tiempo, la nueva situación ha iniciado una serie de acciones proambientales y oportunidades económicas sostenibles tales, que los propios conceptos de desarrollo o de bienestar se han visto cuestionados y redefinidos. Este fenómeno se puede explicar, en parte, gracias al hecho de que la mayoría de las políticas de conservación de aves no representan una amenaza sustancial al crecimiento. Al contrario, estas políticas están en la base de muchas estrategias que buscan una forma diferente de crecimiento o comenzar nuevas oportunidades de crecimiento donde otras posibilidades, industriales o turísticas, no resultaban adecuadas. No obstante, la industria de las aves silvestres se está convirtiendo en un elemento básico de un nuevo tipo de desarrollo basado en una economía más cualitativa, no-material, orientada a los servicios y al ocio, y donde no sólo el conocimiento, sino también la buena diversión y el recreo cultural natural son factores cruciales en la nueva orientación de los actuales sectores de producción y consumo.

Asimismo, la referencia a los pájaros parece adoptar un carácter irresistible en el campo de la educación de la ciencia ambiental y natural para un enorme sector del público en general. Una posible explicación a este fenómeno es que las aves silvestres representan la parte más accesible de lo que ha quedado de la «naturaleza salvaje». Más aún, y como defiende el historiador William Cronon, pueden simbolizar la conciencia de la autonomía de la naturaleza nohumana y que queda representada en la naturaleza salvaje, lo cual es susceptible de propiciar conductas ambientalmente responsables que permitan corregir la "arrogancia humana» ${ }^{30}$. De igual forma, las aves y las actitudes que sostienen su conservación parecen constituir una introducción muy poderosa al pensamiento sistémico y al conocimiento ecológico, lo que permite al público en general desentrañar las conexiones ambientales de las sociedades humanas con su entorno, el conocimiento del cual, de otra forma, sería muy difícil de obtener; o aún menos de comprender a través de un esfuerzo puramente racional o mediante la exposición de las complejas explicaciones de carácter científico $^{31}$. La observación de las aves normalmente combina una actividad al aire libre, habitualmente agradable, con una serie de incentivos para apren-

29. A. Giddens (1984).

30. W. Cronon (1996: 24).

31. S. Giner y D. Tábara (1999). 
der cómo interpretar sus interacciones ecológicas. Por ejemplo, las rutas naturalistas y los centros de interpretación en santuarios para aves, tienden a mostrar a los visitantes no sólo los tesoros naturales del lugar, sino también a producir ejemplos de cómo el impacto medioambiental negativo y la contaminación provocada por el mal crecimiento de las sociedades humanas afecta a los hábitats de los pájaros. Una vez estas ideas se han conectado a la experiencia personal, si es que éste llega a ser el caso, comienza la posibilidad de entrelazar las dos esferas, la humana y la natural. El resultado es la creación de un nuevo tipo de relación entre el mundo humano y el de las aves, que afecta tanto al sujeto como al objeto, y donde, en el caso del primero, puede ser expresado de formas muy diversas. Por un lado, para algunos entusiastas de los pájaros especialmente sensibles, puede tomar la forma de una especie de conversión pseudmística de carácter ecológico, donde las aves pasen a ser consideradas como objetos de reverencia, iconos de la revelación cósmica o fuentes que ayuden a desvelar verdades trascendentales. Mientras que aquéllos menos susceptibles de adoptar una actitud ecorreligiosa, pueden encontrar nuevas razones para involucrarse en el descubrimiento de otras realidades medioambientales científicas, el desarrollo de nuevos conceptos ecológicos o la reunión de datos empíricos para comprobar sus hipótesis «objetivas» al respecto del mundo de las aves $^{32}$. Los pájaros silvestres han llegado a constituir una fuente de inspiración espiritual, un objeto de consideración moral y, al mismo tiempo, una forma de conocimiento sistémico del mundo natural y humano.

Es difícil de creer que la nueva situación de comprensión selectiva, reevaluación y reunificación con la naturaleza hubiera surgido si no hubiera sido gracias al componente arracional y poderosamente simbólico de los mensajes incuestionados acerca de la consideración que las «aves son naturaleza», y que «la conservación de aves es cultura». Evidentemente, los pájaros no son los únicos objetos naturales de la naturaleza, la conservación de aves no es la única expresión de avance cultural ni de relación cultural-natural en la que se pueden observar procesos similares exitosos. Las montañas, los árboles y otros objetos naturales, como algunos mamíferos marinos, fueron también algunos de los primeros en ser seleccionados como medios de comunicación para transmitir el complejo alegato para la conservación o para representar socialmente los ideales y las amenazas que se perciben sobre las identidades nacionales ${ }^{33}$. Lo que es sustancial-

32. Por ejemplo, los pájaros y, en particular, los famosos pinzones de las Galápagos, se dice que inspiraron a Darwin su teoría de la evolución y se muestran por doquier en láminas de los libros de texto y en exhibiciones de historia natural para ilustrar tal evento. Pero, como Frank J. Sulloway ha señalado, Darwin no descubrió su teoría a través de la observación de los pinzones ni esa especie ha contribuido en gran manera (y más que probablemente, en pequeña manera) al desarrollo de sus ideas. De hecho, los pinzones ni siquiera se mencionaban en el Origen de las especies y "Darwin no hizo absolutamente ningún esfuerzo mientras estaba en Galápagos para clasificarlos por islas» (F. J. Sulloway, 1982: 39). Para un texto de sociología ambiental que recree este mito basado en el trabajo de David Lack, Darwin's Finches (1947), ver C. R. Humphrey y F. R. Buttel (1982: 36-39).

33. E. Darier y D. Tábara (2001). 
mente diferente en la actualidad es la intensidad que los medios globales de comunicación de masas, y los políticos y conservacionistas de todo tipo repetidamente capturan imágenes de pájaros y utilizan a las aves en sus discursos a la hora de referirse a la pureza, a la contaminación o a los peligros que acechan al medio ambiente. Además de ello, las aves se han convertido en un poder nuevamente redescubierto de educación medioambiental, y que otros objetos naturales no parecen haber podido asumir. De este modo, una y otra vez, los pájaros silvestres se utilizan, en muchas ocasiones de forma inconsciente, como una referencia incuestionable en la noción actual de naturaleza. Aquellos medios de comunicación o discursos políticos que muestran su apoyo a la conservación de las aves tienen grandes posibilidades de ser recompensados con carisma y prestigio, mientras que sus oponentes serán castigados con el estigma del salvajismo y la reprobación social ${ }^{34}$. A pesar de ciertas resistencias que aún existen, los discursos donde se señala que los pájaros son naturaleza y que la conservación de aves es cultura parecen haberse convertido en uno de los más celebrados y aceptados presupuestos en las sociedades urbanas globales contemporáneas ${ }^{35}$.

\section{Conclusión}

Las sociedades contemporáneas urbanas informacionales, altamente mediadas por la televisión y energéticamente intensivas, se enfrentan a dos tipos de límites en relación con las cuestiones ambientales y de sostenibilidad. Los primeros se relacionan con el impacto negativo e irreversible sobre la base ecológica y natural de la expansión de las poblaciones humanas, el creciente nivel de riqueza per cápita y el tipo de tecnología estructural utilizada. Los otros límites son de tipo social y cultural, y se refieren a la capacidad de esas sociedades de percibir, aprender y actuar debidamente y a tiempo para revertir la tendencia negativa actual del cambio medioambiental. Desde un punto político y educacional, las aves tienen un enorme potencial ambiental que tan sólo ahora está comenzando a reconocer. Al ser las aves una de las especies animales en libertad más familiares, identificables y extendidas que habitan en áreas más cercanas a los núcleos humanos, las conexiones y los impactos ecológicos de las actividades humanas en los hábitats naturales restantes pueden ser fácilmente percibidos y experimentados. Permiten, por ejemplo, aprehender una visión del universo natural como un sistema total y único, en continuo movimiento - migratorio—, en el cual las divisiones políticas y administrativas

34. Los desastres de fugas de petróleo, cuyas imágenes se repiten constantemente en la televisión en forma de aves empapadas en crudo. Sin embargo, como fue en el caso de la invasión iraquí de Kuwait, algunas personas interpretan que esta estrategia quizás no tenga como objetivo latente el informar al público sobre las consecuencias ambientales de la guerra o del consumo mundial de petróleo, sino más bien podría situarse en la línea de la sentencia de William Blake, donde Sadam Husein sería el que «querido será jamás por el hombre».

35. Para una visión más en profundidad de la idea del éxito en los discursos o problemas medioambientales, ver J. Hannigan (1995). 
carecen de sentido. Por ello, las actividades de conservación de avifauna, en su función de señalar nuevas verdades y realidades ambientales a amplios sectores del público, pueden resultar decisivas en el presente momento a la hora de abordar algunos de los más complejos límites culturales a la sostenibilidad ${ }^{36}$. Las formas de vencer algunas de estas barreras culturales pueden ser complejas, y posiblemente demanden un tipo diferente de comunicación y de educación de amplios sectores de la población al respecto. En lugar de concentrarse tan sólo en el enfoque científico de descripción y utilización utilitarista de la naturaleza y/o del medio ambiente, quizás se necesite una perspectiva más arracional y emocional (que no significa irracional), que permita al público en general el formar parte de los procesos de definición, de puesta en marcha y disfrute del proyecto de la sostenibilidad ${ }^{37}$. Y, en esta línea, la propuesta de un movimiento de conservación de las aves parece aportar un sentido a la acción personal y colectiva que otros movimientos más institucionalizados, jerarquizados y con menos posibilidades de recompensas visibles a la participación, como los de carácter político-formal, difícilmente pueden estimular. Sólo para dar un ejemplo de ello, baste decir que el número de miembros de la RSPB (Royal Society for the Protection of Birds) era unas cinco veces superior a la del partido laborista en el poder en Gran Bretaña durante la segunda legislatura de Toni Blair.

El movimiento internacional de conservación de las aves refleja, así, el éxito de un enfoque concreto de la cultura occidental en la selección, comprensión y reunificación con la naturaleza. Se trata del resultado de la confluencia, alianza e interdependencia de dos proyectos históricos. Ornitólogos y entusiastas no expertos, principalmente en países anglosajones, intentaban desarrollar dos relaciones distintas con el mismo objeto natural, pero ninguna de las dos comunidades podía alcanzar sus metas sin la ayuda de la otra. En la actualidad, los medios de comunicación de masas, la industria turística transnacional y una multitud de planes locales medioambientales han añadido una nueva dimensión a los significados ya existentes sobre las aves a nivel social, económico y político. En la era global, los pájaros están revelando de manera continua nuevas verdades inesperadas acerca del impacto humano en ecosistemas locales y globales, a lo cual se suma su capacidad de promover un compromiso social de

36. En este contexto, la noción de límites culturales a la sostenibilidad se utiliza para reconocer y enfatizar el hecho de que los sistemas culturales tan sólo se pueden entender parcialmente y, por lo tanto, los límites culturales han de existir. También se pretende con ello poner de manifiesto las limitaciones del discurso y el conocimiento medioambiental científico, así como el potencial para la acción de otros discursos legos e informales sobre la naturaleza, el medio ambiente y la sostenibilidad. Partiendo de la base de que siempre existen límites culturales para generar y comunicar el conocimiento medioambiental, no es posible encontrar un tipo de conocimiento que pueda eliminar completamente la ignorancia, vencer la indecisión o manejar con total control las cuestiones sobre complejidad y sostenibilidad.

37. Sí a una perspectiva arracional, pero no irracional. En este sentido, y teniendo en cuenta sus efectos en el momento de mejorar los estándares de sostenibilidad y desde el punto de vista ecológico (y carismático), es «racional» el querer y admirar a las aves silvestres (S. Giner y D. Tábara, 1999, y D. Tábara, 1999). 
gran calado por el medio ambiente entre el público en general. La avifauna se ha convertido en una fuente para el descubrimiento de muchas realidades medioambientales, y sus hábitats, los espacios para encontrar y recrear en éstos en objetos animados y cuasi autónomos de la vida humana, una especie de revelaciones de tipo religioso, la fuente de símbolos mediados de estigma o de prestigio, así como un incontestable indicador de calidad ambiental. Por añadidura, estas nuevas culturas-naturalezas híbridas están alcanzando una extensión mundial (a pesar de algunas resistencias), en la forma de un nuevo internacionalismo ecológico. Algunas personas pueden interpretar que estas acciones para proteger a las aves silvestres representan tan sólo una expresión cultural para compensar o minimizar la ansiedad social provocada por la evidente destrucción que las sociedades urbanas están llevando a cabo, tanto en entornos naturales locales como a nivel global. Sin embargo, los hechos revelan que cuando los grupos de conservación de aves han sido capaces de alcanzar sus metas, también han arrastrado con ellas a muchas otras medidas y políticas de carácter proambiental que probablemente no habrían sido adoptadas si no hubiera sido gracias al contenido, enormemente simbólico, emocional y arracional (aunque no irracional) de las evocaciones que se desprenden de las aves silvestres.

Habiendo señalado todo esto, el discurso de conservación de las aves no está, evidentemente, exento de limitaciones, riesgos y contradicciones. Es obvio que las aves no son la única naturaleza, ya que las aves constituyen una especie más entre otros muchos objetos naturales que pueden resultar de utilidad o ser intrínsecamente importantes en las relaciones de los seres humanos con la naturaleza. Las reservas naturales de aves tienden a establecer las fronteras de los sistemas ecológicos de acuerdo con la presencia o ausencia de especies concretas especialmente relevantes desde el punto de vista de su interpretación social, científica o estética, pero a menudo tienden a pasar por alto muchas otras relaciones sistémicas que pueden ser igualmente importantes para la compresión de las conexiones medioambientales humanas y no-humanas de ámbito tanto local como global. Si la sociedad ha de tomar conciencia plena de los problemas y desarrollar los compromisos sociales necesarios para enfrentarse al cambio ambiental, muchos otros objetos naturales y relaciones deberán ser tenidos en cuenta con igual intensidad por parte del público en general. Enfatizar en demasía la importancia de proteger las aves, como si asi se estuviera protegiendo la naturaleza como un todo, puede crear la ilusión de sostenibilidad en situaciones donde, de hecho, la necesidad de reparar el impacto global medioambiental causado por el crecimiento económico global requiere mucho más que la creación de unas pocas reservas naturales para aves. Aunque este objeto natural, familiar y lleno de significado pruebe ser muy efectivo como introducción y para despertar el interés del público por la educación y el compromiso con las cuestiones de la sostenibilidad, es obvio que por sí mismo no es suficiente.

Finalmente, desde el comienzo de la presente contribución, se ha defendido la idea de que existen tantas «naturalezas» como relaciones sociales con la naturaleza. Sin embargo, en este texto se rechaza, asimismo, la idea simplista de que toda la naturaleza es cultura, con lo que se priva de todo contenido a la 
naturaleza ${ }^{38}$. De esta forma, no apoyo tampoco los presupuestos de los constructivistas radicales de que todos los límites naturales son de índole cultural. En contraste con esta idea, aquí se defiende la existencia de ambos tipos de límites dentro de las relaciones sociales con la naturaleza: límites para comprender y racionalizar el cambio ambiental actual y límites al crecimiento de las sociedades humanas continuando con el patrón actual caracterizado por el alto consumo energético, la destrucción de las diversidades y un modo de desarrollo altamente contaminante. Tanto los límites culturales como los ecológicos confieren la forma y el contenido de nuestras relaciones con la naturaleza y, en particular, de las oportunidades de construir relaciones más sostenibles en un futuro cercano. En el presente artículo, se ha pretendido señalar que algunas criaturas vivientes, la forma y el contenido de las cuales a menudo damos por supuesto, y que precisamente muestran un alto poder de evocación como es el caso de las aves silvestres, pueden ayudar a las sociedades urbanas globales a ser más conscientes y a comprometer grandes sectores de la población en la superación o la gestión de ambos tipos de límites a la sostenibilidad.

\section{Agradecimientos}

Este artículo se benefició de las conversaciones mantenidas en su día con Eric Darier, Salvador Giner, Bernd Kasemir y Fiona Thomas. Dedico esta versión actualizada a Martí Boada, de la Universidad Autónoma de Barcelona, quien bien entiende la práctica y el sentido de los argumentos aquí esgrimidos.

\section{Bibliografía}

Atterborough, D. (1998). The Life of Birds. Princenton: Princenton University Press. Barrow, M. V., Jr. (1998). A Passion for Birds. American Ornithology after Audubon. Princenton: Princenton University Press.

Buell, L. (1995). The Environmental Imagination: Thoureau, Nature Writing, and the Formation of American Culture. Cambridge: Harvard University Press.

Carson, R. (1962). «And No Birds Sing». En: Silent Spring. Londres: Hamish Hamilton, p. 84-105.

Charles, L.; Dogde, J.; Milliman, L.; Stockley, V. (1995). «Where you at? A Bioregionalist Quiz». En: CRONON, W. (ed.) (1995). Uncommon Ground. Rethinking the Human Place in Nature. Nueva York: W. W. Norton.

Cronon, W. (1996). «The Trouble with Wilderness; or Getting Back to the Wrong Nature». Environmental History, 1(1): 7-28.

Crosby, A. W. (1986). Ecological Imperalism: The Biological Expansion of Europe 9001900. Cambridge: Cambridge University Press.

DARIER, E.; TÁBARA, D. (2001). «Natural Objects and National Identity. Mountains in Catalonia and Quebec». Ponencia presentada en el II Congrés Català de Sociologia. Traducido al Catalán como «Els objectes naturals i la identitat nacional. Les muntanyes a Catalunya i al Quebec». Papers. Revista de Sociologia, 82. 
Doughty, R.W. (1975). Feather Fashions and Bird Preservation. Berkely: University of California Press.

Drouin, J.M.; Bensaude-Vincent, B. (1996). «Nature for the people». En: Jardine, N.; SECORD, J.A.; SPARY, E.C. Cultures of Natural History. Cambridge: Cambridge University Press.

Evernden, N. (1992). The Social Creation of Nature. Baltimore: The John Hopkins University Press.

FARBER, P.L. (1997). Discovering Birds. The Emergence of Ornithology as a scientific Discipline: 1760-1850. Baltimore: The John Hopkins University Press.

Giddens, A. (1984). The Constitution of Society. Cambridge: Polity Press.

GINER. S.; TABARA, D. (1999). "Cosmic Piety and Ecological Rationality». International Sociology, vol. 14(1): 59-82.

Hannigan, J.A. (1995). Environmental Sociology. A Social Constructionist Perspective. Nueva York: Routledge.

HiLl, J. (1997). An Exhilaration of Wings. The Literature of Birdwatching. Nueva York: Viking.

HumPHREY, C.R.; ButTel, F.R. (1982). Environment, Energy, and Society. Belmont, California: Wadsworth.

KASTNER, J. (1994). «Long Before Furs, It Was Feathers That Stirred Reformist Ire». Smithonian, 25 (julio): 96-104.

Latour, B. (1993). We Have Never Been Modern. Southampton, Gran Bretaña: Harvester Wheatsheaf.

MACNAGHTEN, P.; URRY, J. (1995). «Towards a Sociology of Nature». Sociology, 28(2): 203-220.

MaluQuer i Sostres, J. (1986-1993). «Ornitologia». En: Gran Enciclopèdia Catalana. Barcelona: Enciclopèdia Catalana.

MarX, L. (1964). The Machine in the Garden: Technology and the Pastoral Idea in America. Nueva York: Oxford University Press.

NASH, R.F. (1989). The Rights of Nature. Madison, Wisconsin: University of Wisconsin Press.

Mitchell, R.W.; Thompson, N.S.; Miles, H.L. (1997). Anthropomorphism, Anecdotes and Animals. Nueva York: State University of New York Press.

PAPPS, S.(2001). "The Killing Fields». Birdwatch, 106 (abril): 26-29.

ROYAL SOCIETY FOR THE PROTECTION Of BiRDS (2000). «Annual Report». Birds, 18(3). Bedfordshire: RSPB.

SHACKLETON (1962). «Introduction». En: CARSON, R. Silent Spring. Londres: Hamish Hamilton, p. XIII-XIX.

STReSEMANn, E. (1975). Ornithology from Aristotle to the Present. Cambridge: Harvard University Press.

SullowAY, F. (1982). «Darwin and His Finches: The Evolution of A Legend». Journal of the History of Biology, 15: 1-53.

TÁbara, D. (1999). Acció Ambiental. Aprenentatge i participació vers la sostenibilitat. Binissalem, Illes Balears: Di7 Edicions y Associació Catalana d'Educació Ambiental. Associació Balear d'Educació Ambiental.

Thomas, K. (1983). Man and the Natural World. Nueva York: Pantheon Books.

Thoreau, D. (1993). Thoreau on birds. Boston, Mass: Beacon Press. [1910]

The Gallon Environment Letter (2001). «Bird Watching. A US \$25 Billion a Year Industry». The Gallon Environment Letter, vol. 5 (5), 6 de febrero. 